\section{MAY 2000}

- 2nd annual North American softwood conference, 1-3 May.

Quebec City, QC, Canada. Contact: Christina Castagnaro, Quebec Wood Export Bureau, 2nd Annual North American Softwood Conference, 979, avenue de Bourgogne, Bureau 540, Saint-Foy, Quebec, Canada G1W 2L4. Tel: 418-650-6385; Fax: 418-650-9011; e-mail: infor@Quebecwoodexport.com

- 41 st Canadian Wood Council annual convention, 3-5 May.

Quebec City, QC, Canada. Contact: Suzanne Duguay, Tel: 613-747-5544; e-mail: sduguay@cwc.ca

- Mediterranean silviculture with emphasis on Quercus suber, Pinuspinea and Eucalyptus (IUFRO), May. Spain. Contact: Gregorio Montero, Tel: 341-347-6854; Fax: 34-1-357-6859

- The future of the Mediterranean rural environment: prospects for susfainable land use and management, 8-11 May.

Menemen, Turkey. Contact: Gill Burrows, Cranfield University, Silsoe, Bedfordshire, MK45 4DT, UK.; Fax: 44-1525-863-344; e-mail: gburrows@cranfield.ac.uk

- The role of boreal forests and forestry in the global carbon budget, 8-12 May. Edmonton, Alberta, Canada. Contact: Carbon Conference Coordinator, 5320-122 Street, Edmonton, Alberta T6H 3S5; Fax: (780) 435-7365; e-mail: carbon@nofc.forestry.ca; Web Site: www.nofc.forestry.ca/carbon

- Canada's forest sector in the millennium year, 14-18 May.

Thunder Bay, Ontario. Contact: Frances Bennett-Sutton, Tel: (807) 622-8228; email: fsb2000@flash.lakeheadu.ca or Dr. Ed Iwachewski, Tel: (807) 343-4016, email: ed.iwachewski@mnr.gov.on.ca. Website: http://www.forconfor2000.org.

- 5th meeting of the conference of the parties to the convention on biological diversity, 15-26 May.

Nairobi, Kenya. Contact: CBD Secretariat, World Trade Center, 393 Jaques St., Suite 300, Montreal, Quebec Canada H2Y 1N9: Fax: (514) 288-6588; e-mail: chm@biodiv.org; Web: www..biodiv.org

- Issues in world forestry and forest products: tropical study program in
Nicaragua, 15 May-3 June.

Laguna de apoyo, Nicaragua and Gamboa, Panama. Contact: A.L. Hammett, College of Natural Resources, 210 Cheatham Hall, Virginia Tech, Blacksburg, Virginia 24061, USA. Fax: (540) 231-8176; e-mail: himal@vt.edu

- Improvement of wood quality and genetic diversity of oaks "Oak 2000" (IUFRO, Div. 1), 20-25 May.

Zagreb, Croatia. Contact: Heinrich Spiecker, Institute for Forest Growth, Univ. of Freiburg, Bertoldstr. 17, D-79085, Freiburg, Germany.e-mail: instww@ruf.uni-freiburg.de

-43rd annual conference of the International Association of Great Lakes Research (IAGLR), 21-26 May.

Cornwall, Ontario, Canada. Contact: Christina Collard, St. Lawrence Rivers Institute of Environmental Sciences, 1111 Montréal Road, Cornwall, Ontario K1H 1E1. e-mail: ccollard@riverinstitute.com; Web site: http://www.riverinstitute.com

- ASPRS, the Imaging and Geospatial Information Society 2000 annual conference, 22-26 May.

Washington, DC. Contact: ASPRS, Fax; 410-641-8341; e-mail: wboge@aol.com

- Sustainable forestry management in Africa in the 21 st century, 22-27 May. Abuja, Nigeria. Contact: Dr. Stephen A. Dada; e-mail: dadest@mail.skannet.com; Website: www.foresters.org/anword/ conference.htm\#top

- 3rd AGILE conference on geographic information science (AGIL 2000), 25-27 May.

Helsinki/Espoo, Finland. Contact: http://www. gi.fi/agile2000/

- Impacts of air pollution and climate change in forests-19th. In't I Meeting for specialists in air pollution effects on forests (Div. 7, IUFRO), 27-30 May. Houghton, Michigan. Contact: David Karnosky; Fax: (906) 487-2897; e-mail: karnosky@mtu.edu

- 6th symposium and workshop, Canadian Society for Landscape Ecology and Management, 31 May-2.

Guelph, Ontario. Contact: e-mail: cslem@ uoguelph.ca; Web site: www.uoguelph.ca/ $\sim$ cslem
JUNE 2000

- Caring for our land: stewardship and conservation in Canada, 3-6 June. Guelph, Ontario Canada. Contact: Peter Mitchell: e-mail: claws@uoguelph.ca; Website: www.uoguelph.ca/ claws/conference

- International symposium on the biogeography of Southeast Asia 2000, 4-9 June.

Leiden, The Netherlands. Contact: Rienk de Jong: Fax: 31-71-513-3344; e-mail: jong@nnm.nl

- New knowledge and solutions for forest management, 5-8 June.

Detour Lake, Ontario. Contact: Lake Abitibi Model Forest, Tel: 705-258-4278; e-mail: workshop@lamf.net; Web site: www.lamf.net

- 6th circumpolar symposium on remote sensing of polar environments, 12-14 June.

Yelllowknife, Northwest Territories, Canada. Contact: Web site: www.gov.nt.ca/rived/rs/ circumpolar2000/

- Sustaining aspen in western landscapes, 13-15 June.

Grand Junction, CO, USA. Contact: e-mail: spenconf@cnr.colostate.edu; Website: www.cnr.colostate.edu/outreach/aspen/

- 8th International symposium on society and resource management, 17-22 June.

Bellingham, Washington, USA. Contact: www.ac.wwu.edu/natresco/: John C. Miles: e-mail: jcmiles@nessie.cc.wwu.edu

- 53rd Canadian Water Resources Assocition Annual Conference, 21-23 June. Saskatoon, Saskatchewan, Canada. Contact: Phil Adkins: e-mail: adkinsp@em.agr.ca

- Geoinformatics 2000: International conference on geographic informafion science and fechnology, 21-23 June. Monterey, California. Contact: Dr. Yong Lao; e-mail: geoim2000@monterey.edu; Website: http://www.monterey.edu/geoim2000/

- 20th EARSel Symposium - Remote sensing in the 22st century: A decade of trans-European remote sensing cooperation, 21-23 June.

Dresden, Germany. Contact: Mrs. M. Godefroy: e-mail: earsel@meteo.fr: fax: 33-145567361 
- Canadian Phytopathological Society - Pacific Division of the American Phytopathological Society, 18-21 June. Victoria, BC, Canada. Contact: Dr. Jack Sutherland, Fax:250-598-1959; Tel: 250478-4850; Web site: http://www.uvcs.uvic.ca/ conferce/CPS_aps/

- Ecosummit 2000. Integrating the sciences. Understanding and solving problems in the 21st. century, 18-22 June. Halifax, Nova Scotia, Canada. Contact: Amy Richardson, Fax: 44-1865-843958; e-mail: a.richardson@elsevier.co.uk; Website: www.elsevier.com/locate/ecosummit

- Science and technology for the new millennium (ASCE Watershed Management 2000 Conference), 21-24 June. Fort Collins, CO, U.S.A. Contact: e-mail: dfrevert@do.usbr.gov

- Wood adhesives 2000 (Div. 5, IUFRO), 22-23 June.

Lake Tahoe, Nevada. Contact: John A. Youngquist; Fax: 608-231-9582; Website: http://www.fpl.fs.fed.us/pdcomp/

- Multipurpose management of mountain forests: concepts, methods, techniques (IUFRO, Task Force Meetings), 25-30 June.

Pralognan-la-Vanoise. Contact: Martin Price, 11 Bevington Road, Oxford 0X2 6NB, U.K. E-mail: martin.price@ecu.ox.ac.uk

- 2000 World conference on natural resource modelling, 26-30 June.

Wageningen, the Netherlands. Contact: Joost Meulenbroek; fax: 31-317-485309; e-mail: joost.meulenbrock@alg.vl.wau.nl; Web site: www.cqs.washington.edu/-gordie/rma/html

- Forestry beyond the timberline, 27-30 June.

Akureyri, Iceland. Contact: Throstur Eysteinsson, Iceland Forest Service, P.O. Box 98, 700 Egilsstadir, Iceland. Fax: 354-471-2172; email: throstur@simnet.is

\section{JULY 2000}

- Watershed 2000 - National and international challenges of managing watersheds, 9-12 July.

Vancouver, BC, Canada. Contact: e-mail: msc@wef.org.

- 4th Infernational symposium on spatial accuracy assessment in natural resources and environmental sciences, 12-14 July.

Amsterdam, The Netherlands. Contact: Fax:
31-20-525-4799; e-mail: accuracy@frw.uva.nl; Website: www.gis.wav.nl/accuracy2000/

- The impact of global environmental change on forests, and the impact of forests on global environmental change, 16-21 July.

Mérida, Yucatan, México. Contacts: Eric Diaz M., Dir. For., INIFAP, Km. 24 Carretera Mérida-Motul, Mococcha, Yucatan CP 97454, México. e-mail: era@cirse.inifap.conacyt.mx: CANADA - Judy Loo, Canadian Forest Service, Box 4000, Fredericton, NB E3B 5P7: e-mail: jloo@nrcan.gc.ca

- Geoinformation for all, XIX Congress of the International Society for Photogrammetry and Remote Sensing (ISPRS), 16-23 July.

Amsterdam, The Netherlands. Contact: Congrex Holland BV, Box 302, $1000 \mathrm{AH}$, Amsterdam. Fax: 31-20-50-40-225; e-mail: isprs@congrex.nl

- IGARSS 2000: International geoscience and remote sensing symposium, 24-28 July.

Honolulu, Hawaii, USA. Contact: IEE Geoscience and Remote Sensing Society, 17906 St. Emilion Court, Spring, TX 77379, USA. Fax: 281-251-6068; e-mail: tstein@phoenix.net

- World conference on timber engineering, 31 July-3 August.

Whistler, BC, Canada. Contact: Fax: (604) 681-2503; e-mail: congress@venuewest.com

\section{AUGUST 2000}

- Bamboo 2000 international symposium, 2-4 August.

Chiang Mai, Thailand. Contact: Faculty of Forestry, Kasetsart University, Bangkok 10900 Thailand; Fax: 66-2-942-8112; e-mail: fforlwp@nontri.ku.ac.th
- Tropical forestry research: Challenges in the new millennium, 2-4 August.

Peechi, Kerala State, India. Contact: J.K. Sharma, Kerala Forest Research Institute, Peechi - 680 653, Kerala, India. Fax: 91-487782249; e-mail: libkfri@md2.vsnl.net.in

- Management of juniper forests: looking for methods, techniques and solutions, 6-11 August.

Osh, Kyrgzstan. Contact: Irina Unusova, LES - IC, Box 2011, Central Post Office, Bishkek, 720000, Kyrgyzstan, CIS. e-mail: irina@lesic.elcat.kg

\section{- XXI IUFRO World Congress, 7-12} August.

Kuala Lumpur, Malaysia. Contact: Organizing Committee, Forest Research Institute, Malaysia, Kepong, 52109 Kuala Lumpur, Malaysia. Fax: 60-3-6367753; e-mail: iufroxxi@frim.gov.my; Web site: http://frim. gov.my/iufro.html

\section{- Data collection in the tropics (IUFRO,} Div. 4), 7-12 August.

Kuala Lumpur, Malaysia. Contact: Mohammed Ellatifi, Service des Eaux et Forêt, PB 12507 Casablanca, Morocco. e-mail: m.ellatifi @mailcity.com

- CIF 2000 - Annual conference of the Canadian Institute of Forestry, 6-10 August.

Corner Brook, Newfoundland. Contact: Len Moores, Fax: 709-637-2290.

- Western international forest disease work conference, 14-18 August.

Waikoloa, Hawaii. Contacts: Sue Hagle (Program); Tel: 208-926-4275; e-mail: shagle/rl@fs.fed.us; Local arrangements:

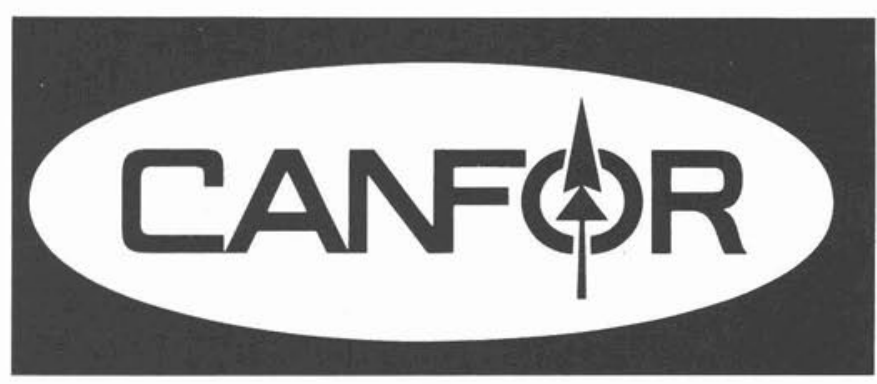

A CIF/IFC Corporate Sustaining Member 
Jerry Beatty; e-mail: jbeatty/r6pnw_mthood@ fs.fed.us; Web site: www.fs.fed.us/foresthealth/ technology/wif/index.html

- Genetic resource management building strategies for the new millennium (Canadian Tree Improvement Association), 15-17 August.

Sault Ste.Marie, Ontario, Canada. Contact: Dennis Joyce: Fax: 704-945-6667; e-mail: dennis.joyce@mnr.gov.on.ca

- 5th international symposium on environmental geotechnology and global sustainable development, 15-21 August.

Belo Horizonte, Minas Gerais, Brazil. Contact: Fax: 55-31-238-1793; URL: http://5ieggsd. eng.ufmg.br

- Forest ecosystems: Ecology, conservation, and sustainable management, 15-21 August.

Chengdu, China. Contact: Dr. Shi Zuomin \& Ms. Dong Na, Chinese Academy of forestry Wanshoushan, Beijing 100091, China. Fax: 86-10-62884972; e-mail: shizm@fee.forestry.ac.cn

- XXI International Congress of Entomology, 20-26 August.

Iguacu Falls, Brazil. Contact: Dr. Décio Luiz Gazzoni, P.O. Box 231, 8f6001-970 Londrina - PR Brazil; Fax 55-43-371-6100; e-mail: iceweb@cnpso.embrapa.br. Web site: www.embrapa.br/ice

4th International symposium on the tree, 20-26 August.

Montreal, Québec, Canada. Contact: Michel Labrecque: Fax: 514-872-9406: e-mail: arbre2000@ville.montreal.qc.ca; Web site:http://www.irbv.umontreal.ca/arbre2000tree 2000

- Disfurbance dynamics in boreal forests, 21-25 August.
Kuhmo, Finland. Contact: Timo Kuuluvainen; e-mail: kuuluvai@ladybird.helsinki.fi; Web site: http://honeybee.helsinki.fi/dist2000.

- 22nd Canadian symposium on remole sensing, 21-25 August.

Victoria, BC, Canada. Contact: Fax: 613-234 9039; e-mail: casi@casi.ca

- Streamlining local-level information for sustainable forest management, 28-30 August.

Vancouver, BC. Contact: Prof. John L. Innes; Fax; 604-822-9106; e-mail: innes@interchg.ubc.ca; Web site: www.forestry.ubc.ca/ workshop/index.html

\section{SEPTEMBER 2000}

- The 48th Annual meeting of the Western International Forest Disease Work Conference, September (dates, TBA).

Hilo, Hawaii. Contact: Jerry Beatty, USDA Forest Service, 16400 Champion way, Sandy, Oregon 97055; Fax: 503-668-1423; e-mail: jbeatty/r6pnw_mthood@fs.fed.us

-4th International conference on integrating geographic information systems (GIS) and environmental modeling, 2-8 September.

Banff, Alberta, Canada. Contact: e-mail: gisem@colorado.edu; Web site: www. colorado.edu/research/cires/banff

- Opportunities for the new millennium, 4-6 September.

Cairns, Queensland, Australia. Contact: e-mail: killind@prose.dpi.qld.gov.au; Web site: http://www.afg2000.com/

- Models for the sustainable management of temperate plantation forests, 7-9 September.

Bordeaux, France. Contact: Jean-Michel Carnus: e-mail: carnus@pierroton.inra.fr;

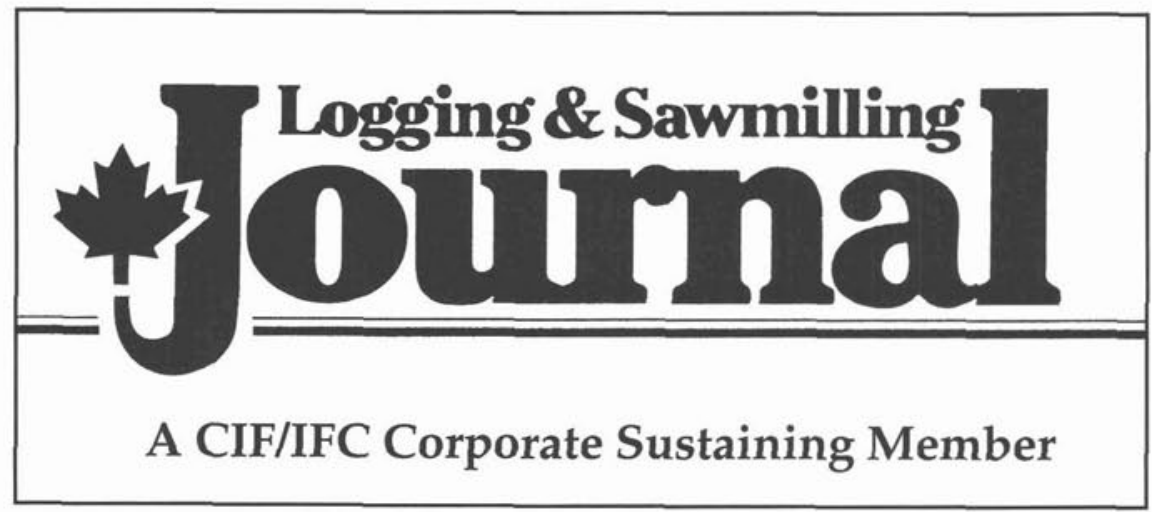

- 2nd International conference GIS for earth science applications, 11-14 September.

Menemen, Turkey. Contact: Anadola University: Fax: 90-222-323-9129; e-mail: cgesa@anadolu.edu.tr; Web site: http://icsesa. anadolu.edu.tr

-3rd interantional symposium - Application of remote sensing in forestry, 12-14 September.

Zvolen, Slovak republic. Contact: Lubomir Scheer: Fax: 421-855-5332654; e-mail: scheer@vsld.tuzvo.sk

- Kuratorium fur Waldarbeit und Forsttechnik e.V. (KWF) Conference 2000, 13-17 September.

Celle, Germany. Contact: KWF Central Unit, Tel: 49-6078-785; Fax: 49-6078-78539; e-mail: kwf.info@t-online.de; web site: http://www.dainet.de/kwf/kwf.htm

- Demo 2000 International, 12-16 September.

Kelowna, BC, Canada. Contact: Wayne Novak, Activexpo. Tel: (514) 426-4010; Fax: (514) 426-5073; e-mail: wnovak@colba. net

- 12th infernational symposium on non-destructive festing of wood, 13-15 September.

Sopron, Hungary. Contact: Ferenc Divos: email: divos@efe.hu; Web site: http://sun30. efe.hu/ndt2000/

- Trends 2000: 5th international outdoor recreation \& tourism trends symposium, 17-20 September.

Lansing, Michigan, USA. Contact: Randy Alampay: e-mail: alampayr@pilot.msu.edu

- Fragmentation 2000-sustaining private forests in the 21 st century, 18-20 September.

Annapolis, Maryland, USA. Contact: Terri Bates: e-mail: bates-stasny@erols.com

- New approaches to the management of neotropical primary rainforests by industries and communities, 20-22 September.

Belém, Brazil. Contact: Dr. Natalino Silva, Brazilian Agricultural Research Corp., CP 48 , CEP 66240, Belem, Brazil; Fax: 5591-226-9845; e-mail: natalino@cpatu. embrapa.br

- Monitoring and modeling catchment water quantity and quality, 27-29 September. 
Gent, Belgium. Contact: e-mail: erb2000@ neiden.rug.ac.be; Web site: http://neiden.rug. ac.be/erb2000

- IPC 2000 - 21 st session of the International Poplar Commission, 24-30 September.

Portland, Oregon. Contact: Jim Richardson, 1878 saunderson Drive, Ottawa, ON, Canada, K1G 2C5; Fax: 613-521-1997; e-mail: jrichardson@on.aibn.com Websites: www. ncfes.umn.edu/ipc2000/: www.poplar.ca

- 7th International inorganic-bonded wood and fiber composite materials conference, 25-27 September.

Sun Valley, Idaho, USA. Contact: Al Moslemi, College of Forestry, University of Idaho, Moscow, ID 83844-1132; e-mail: amoslemi @novell.uidaho.edu

- Woody biomass in energy production in Europe, 25-27 September.

Joensuu, Finland. Contact: Ms. Brita Pajari: Fax: 358-0-13-124-393; e-mail: brita.pajari @efi.fi

- SAF symposium on systems analysis in forest resources, 27-30 September. Aspen, Colorado: Contact: www.for.msu.edw/ e4/ssafr2000.htm

\section{OCTOBER 2000}

- Montaine alternative silvicultural systems (MASS) workshop: integrating research and operations, 3-5 October. Courtenay, British Columbia. Contact: Tom Hedekar, Malaspina University-College Forestry Extension Program; Tel: 250741-2597; Fax: 250-741-2190; e-mail: tom.hedekar@mala.bc.ca; Web site: www. mala.bc.ca/www/forestex/index.htm

- Elmia Timber - International wood products and components trade fair, 4-7 October.

Jonkoping, Sweden. Contact: Alan Sherrard, Fax: 46-36-16-46-92; e-mail: timber@ elmia.se; Website: www.elmia.se/timber

\section{- Forest genetics for the next millennium (IUFRO), 8-13 October. \\ Durban, South Africa. Contact: Peter Roberts, P.O. Box 100281, Scottsville 3209, South Africa. Fax: 27-331-68905; e-mail: sieg@icfr. unp.ac.za}

- Bio Agro Contact 2000, 15-17 October. Québec, Canada. Contact: Julie Vallières, Fax: 514-521-3193; e-mail: groupexpo@mlink.net;
- Issues in environmental pollution (IEP) 2000, 16-18 October.

Lisbon, Portugal. Contact: Gill Heaton; Fax: 44-0-1865-375855; e-mail: gill.heaton@ virgin.net

- 3rd European conference on applied climatology (ECAC2000), 16-20 October. Pisa, Italy. Contact: Antonietta Falchi, Via Caproni n.8, 50145 Florence, Italy. Fax: 39055-308910; e-mail: falchi@sunserver.iata.fi.cnr.it

- 2nd infernational symposium in new technologies for environmental monitoring and agro-applications, $18-20$ October.

Tekirda, Turkey. Contact: Dr. Feliz Sunar: Fax: 20-212-5737027; e-mail: fsunar@srv.ins. itu.edu.tr

- International conference on multifunctional landscapes, 18-29 October. Roskilde, Denmark. Contact: Jesper Brandt: Fax: 45-46-74-3032.e-mail: gunther@ruc.dk; Web site: www.geo.ruc.dk/ulb/conference.htm

- First international conference on wood in world rivers, 23-27 October.

Corvallis, Oregon. Contact: Stan Gregory, Oregon State University, Corvallis, Oregon: Fax: 541-737-3590; e-mail: stanley.gregory @ orst.edu; Website: http://riverwood.orst.edu

\section{NOVEMBER 2000}

- National convention, Society of American Foresters, 16-21 November.

Washington, DC. Contact: Mike Murphy, SAF, 5400 Grosvenor Lane, Bethesda, MD. 208142198. Tel: 301-897-8270; Fax: 301-897-3690; e-mail: murphym@safnet.org

- Internatonal symposium on GEO environmental recalamation, 20-22 November.

Nagpur, India. Contact: Fax: 91-712-583237; e-mail: rknintsy@webginn.com

Annual conference, International Society of Tropical Foresters, 22 November.

Washington, DC. Contact: Dr. Warren T. Doolittle, President, ISTF, 5400 Grosvenor Lane, Bethesda, MD 20814: Fax: 1-301897-3690; e-mail: istfr@igc.apc.org; Web site: www.cof.orst.edu/org/istf

\section{DECEMBER 2000}

-5th Pacific Rim bio-based composites symposium, 10-13 December.

Canberra, ACT, Australia. Contact: Dept. of Forestry, Australian National University,
Canberra ACT 0200, Australia. e-mail: bio.symposium@anu.edu.au

\section{JANUARY 2001}

- Landscape legacies: managing landscapes in the context of human history and ecosystem change, 8- 10 January. Durham, North Carolina. Contact: Susan Fox; Tel: 919-513-3331; e-mail: sfox@ unity.ncsu.edu

\section{APRIL 2001}

- 16TH Commonwealth Forestry Conference, 18-25 April.

Fremantle, Perth, Western Australia. Contact: Commonwealth Forestry Association, Oxford Forestry Institute, South Parks Rd., Oxford OX1 3RB, UI; Fax: 44-1865-275074; e-mail: cfa@plants.ox.ac.uk

\section{JUNE 2001}

- FAO/ECE/ILO workshop on new developments of wood harvesting with cable systems, June (dates to be announced).

Austria (city to be announced). Contact: R, Heinrich, Forest Products Division, FAO, Viale delle Terme di Caracalla, 00100 Rome, Italy; Fax: 39-06-5705 5137; e-mail: forestharvesting@FAO.org

- Elmia Wood 2001 - International forestry trade fair, 6-9 June.

Jonkoping, Sweden. Contact: Torbjorn Johnsen; Fax: 46-36-16-46-92; e-mail: wood@elmia.se; Website: www.wood.elmia.se

International conference on ex situ and in situ conservation of commercial tropical trees, 11 - 13 June.

Yogyakarta, Indonesia. Contact: Ms Soetitah S. Soedojo; Fax: 62-274-902 220; e-mail: itto-gmu@yogya.wasantara.net.id

\section{SEPTEMBER 2001}

4 49TH Annual Meeting of the Western International Forest Disease Work Conference, September (dates, TBA).

Anchorage, Alaska. Contact: Lori Trummer, USDA Forest Service, 3301 C Street, Suite 522, Anchorage, AK 99503-3956. Fax: 907271-2897; e-mail: ltrummer/r10_chugach @fs.fed.us

\section{SEPTEMBER 2003}

- World forestry congress, 22-30 September.

Quebec City, Quebec, Canada. Contact:

Website: www.wfc2003.org 